\title{
Impediments to Requirement Engineering in Distributed Team
}

\author{
Nabiha Usmani \\ Institute Of Business Administration, Computer Science, Karachi, 75400, Pakistan \\ Email: nabihausmani92@gmail.com \\ Rabbia Hassan \\ Institute Of Business Administration, Computer Science, Karachi, 75300, Pakistan \\ Email: rabbia.hassan91@gmail.com \\ Waqas Mahmood \\ Institute Of Business Administration, Computer Science, Karachi, 75300, Pakistan \\ Email: wmehmood@iba.edu.pk
}

Received: 29 May 2017; Accepted: 13 July 2017; Published: 08 November 2017

\begin{abstract}
Due to the greater availability of skilled software engineers, organizations are increasingly adopting Global Software Development, at relatively lesser costs. Software process in such distributed teams have turned out to be progressively more viable for numerous reasons, due to better communication technologies and maturity level of software industry. However organizations adopting Global Software Development must take into consideration that it is not a risk-free action, as many failures related to it have been reported.

There are a number of challenges when existing tools of globally distributed projects are adopted. Upon deep evaluation of requirement analysis during interactive phase, particular consideration must be given to the requirements of clients and distributed software provider teams which are globally dispersed. In this paper, we present realistic insights gathered from carrying out surveys from IT professionals and people working in the software industry. Moreover, extensive examination of the literature work previously done in this regard is also documented in our paper. The objective of our paper is to get a clear idea about the major factors and challenges faced by the team members of the globally distributed team. After identification of these key factors, based on the results of our survey, we have endeavored to present an analysis on how to overcome these challenges.
\end{abstract}

Index Terms-Requirement gathering, Globally Distributed Development (GSD), Virtual Teams, Offshore Outsourcing, Challenges, Major problems in RE

\section{INTRODUCTION AND BACKGROUND}

Globalization is a key trend in today's business world. Companies are moving forward to operate as if a homogenous worldwide market. Software industry is also influenced by this globalization by allowing multicultural stakeholders to work together in global platform recognized as global software development environment (GSD) (Khan H. H., 2013). The popularity of this environment has provided industry with number of advantages as compared with the traditional software development process (Khan H. H., 2014). The popularity of GSD demands the proper requirement processes which can work for this environment, as the success of a project highly depends on this process (de Gea, 2016). These projects can consist of distributed teams who work together for software project development.

$\mathrm{RE}$ is considered to be the most important activity as it greatly impacts the project outcome. RE is also, known as activity of communication, which can be easily effected in global and distributed environment. Hence special attention is needed in this process. Generally, RE process comprises of five activities: 1) Requirements extraction or elicitation, 2) Requirements analysis and design, 3) Requirements specification, 4) Requirements validation and 5) Requirements management (Kumari, May 2013). Also included are the processes of change request analysis, approving or not approving the changes and implementing approved changes (Minhas N. M., 2014).

Requirement Engineering (RE) is that branch of software engineering discipline that deals with the stakeholders needs supposed to be fulfilled by the software. Before developing a product, 'Requirement' are the prerequisites which needs to be gathered and developed. The first stage of Software development life cycle is RE; that gathers, analyzes, identifies, confirms and documents needs of stakeholders. RE is a crucial activity, any mistake regarding inaccurate or wrong requirement gathering can increase the development cost a lot as compared to original estimation (Ali S. , 2016). Output from this process forms a base for the whole project. RE becomes even more challenging task when e talk about global teams. 
Concept of virtually distributed teams is gaining popularity with time in software industry. Global software development team (GSD) comprises of project's stakeholders belonging to different parts of the world, residing in different countries. Communication is done among the team virtually via email, fax, video calls or any other means of interaction. Gathering of Requirement is the most difficult phase of GSD. RE itself is a challenging domain and when it is done globally it gets complicated. Requirement Change is an unavoidable activity of software development which can occur due to various reasons i.e. due to change in requirements from user, understanding and realization of stakeholders' needs, emergence of new technology. These changes shall be timely managed to achieve successful software development. Requirement change management is demanding in global software development as factors like geographical, social, temporal and cultural differences arise. (Ali N. , Managing requirements change in Global Software Development, 2015) Present techniques and methods do not completely deal with the issues and challenges faced in Global software development GSD. For effective requirement gathering and analysis proper collaboration between stakeholders is vital. It is necessary to educate stakeholders about GSD issues as cultural diversity and language barriers create difficulty for GSD stakeholders. (Ali N., A Method of requirements change management for global software development, 2015).

Since there is a proliferation of distributed projects, it is important that nature of such software development should be understood. Attractive features offered by GSD like large number of skilled workers pool, low salary expense, cheap manual labor, rapid virtual teams' formation etc. causes the organizations to adopt it with lesser hesitation and proper impact analysis. In parallel, some real problems are faced by the teams while working in distributed team environment. As quoted by Aranda etal "distance is the major problem in global software development and it introduces difficulties in globally distributed projects." (Minhas N. M., 2014). One important factor in GSD is deciding what level of correspondence and interaction is necessary between client and development team in each phase. Normally organizations regard phases such as coding and testing (in this client team usually works in an independent manner) as appropriate for off shoring. On the other hand research, has shown that Requirement analysis stress on considerable and constant communication between provider teams and clients. As the communication technologies are getting so successful this supports requirement determination in distributed environment. (Yadav V., 2016)

As Requirement Engineering is highly communicative and collaborative intensive, and also greatly affects other development processes, the practices defined for the process are really important. Most of the traditional rules and principles defined for RE may not help project teams when the setup is globally distributed. With the increase of Global collaboration among the software world, the Requirement Engineering processes have become the key challenge in Global Software Engineering (GSE). Because of the diversity in the background, culture and interests of team members and other stakeholders, a need exists to achieve common understanding among the team regarding to project (Kumari, May 2013).

In global perspective, traditional requirement engineering activities may need to be modified to cater to special cases involved in such setups. The process of requirement elicitation to change analysis may differ and would be needed to adopt according to team's setups and cultures.

The goal of this paper is to identify and enlist the factors during RE process in GSD paradigm. Objective of our paper is to first discuss the literature work done in this regard. Next we have discussed the results of the survey conducted by us. Our target audience for the survey was IT specialist and the people working in the software industry. After the lessons learnt on the basis of survey results the literature has been discussed.

\section{RELATED WORK}

Due to large shift to GSD environment projects, many researches deal with the problems faced by such projects. The teams have tried to stick with old traditional ways of Requirement Engineering in such environment, which not only ended up as a failure of Requirement Engineering process but in many cases a disaster end of project.

Niaziet al (Niazi) suggests that in GSD environment, along with challenges of GSD business nature and culture differences, teams should also focus on the challenges faced by usage of different tools in GSD environment. They list down some of the challenges faced by such teams in using tools for GSD environment. 1) Selection of inappropriate communication tools (synchronous and asynchronous). 2) Problems with adopting and adjusting to suitable tools. 3) Lack of coverage of such tools. 4) Lack of data integration among different tools. 5) Security and privacy issues. 6) Lack of familiarity of tools appropriate for GSD environment. 7) Lack of progress tracking.

Yadavet al (Yadav V. A., 2016) reports a conceptual framework for successful RE process in GSD environment. They have contributed three main factors that need to be focused for successful RE process. 1) Client - Provider roles structure - They have emphasized that there should be a formal control mechanism for close project monitoring. Also, there should be a designated client-side and provider-side coordinator who's assigned and responsible for all communication and coordination needed for RE process. 2) Technologies - Like other researchers, they have also emphasized on the need of using appropriate and effective synchronous communication tools. 3) Process Flexibility - There should be a common framework which will help align teams situated at different locations, with the client. Teams should try to adopt agile principles, iterative approaches, and prototyping to enhance flexibility in processes. With the use of agile processes, the team 
should also be able to deal with incoming change requests with proper process.

Rahmanet al (Rahman, 2015) indicates few other challenges faced in GSD environment and list downs some mitigation strategies, i.e.: 1) Lack of trust among teams can be mitigated through sprint \& iterative planning, as they help to increase trust by making project status visible to everyone. Other than that group activities and co-curricular activities helps team bonding. 2) Skill differences and technical issues. This can be tackled through training for virtual communications, pairing or virtual collaboration. 3) Lack of Visibility \& transparency. This is most important factor in GSD environment that need to be tackled, as it will automatically affect other hurdles. With the use of proper sprint process, regular meetings and appropriate ICT-mediated tools, this can be mitigated.

(Fernández, 2013) In his report presented a generalized and empirical study regarding what is the situation of practices followed in RE. For this purpose, a family of surveys which was globally distributed was designed. Various researchers from different countries worked in collaboration. It started with the initial theory formed from the studies available. To improve the results, survey developed was replicated regularly as a long-term goal so that clear and realistic understanding could be developed on the needs of Requirement Engineering. In this report design of survey families was presented. Survey results started from Germany. The first result from 58 companies of Germany indicated several problems and trends. Out of which most common was that in many companies' artifacts were not properly represented. Inconsistent and incomplete requirements were an important problem experienced by the companies in their projects.

This report provides solution to the problems identified by isolated researches and investigation in this field. The survey's initial phase is based on theory from literature. After that a pilot phase took place to set the validity procedures which could be performed in future. Final results were presented on the basis of survey done in Germany. The first replication is now being done in Netherland. As per the process of replication it comprises of the same questionnaire and infrastructure but the results would be formulated independently. Survey design and results are committed to PROMISE repository. Basically, the objective was to set up a continuously performed replication of survey which could be constantly adapted and results in a proper theory comprising the problems faced in RE.

(Ali S. 2016) In his paper explains the concept of Global software development i.e. GSD. The main objective of the paper is to find the Critical success factors and challenges faced in implementation of requirements. Moreover, practices were found to implement critical success factor as per the requirement implementation model (RIM). For this purpose, methodology of SLR was used. Protocol of SLR was developed for Requirement Implementation Model so that all the challenges faced by the stakeholders while gathering requirements in globally distributed environment through SLR could be listed down. Ali states that his aim was to develop RIM from the perspective of stakeholders and vendors in globally distributed environment. This aim was achieved by finding critical success factor through systematic literature review. Practices and challenges for CSF were searched so that they could be implemented by the approach of questionnaire survey. In the end a proper structure of RIM was proposed.

RIM model proposed in this paper comprises of five stages i.e.

1. Specifying criteria for RIM model development via Literature review and supervisor input

2. Data collection and analysis via SLR, Questionnaire survey

3. Rationalization and structuring of Results via Literature review of various models.

4. Development of RIM model via SLR, Questionnaire survey

5. Evaluation of RIM Model via Case studies.

After this search teams of categories including People, Interference and outcomes of relevance were made to discuss the factors decided. Later procedure and plan for searching was devised. Selection of publication then went through a proper process with an inclusion and exclusion criteria, quality of the papers selected was also assessed on certain criteria. Next step of data extraction was also carried out on the basis of a proper strategy. In the end validation of protocol was achieved by writer's supervisor and his colleague. In this manner, final goal of developing RIM for GSD was achieved.

\section{METHODOLOGY}

The main idea behind our research work is to properly identify the obstacles faced by teams especially in requirement gathering and monitoring, while working in GSD environment. We then analyzed these challenges and came up with the best practices to deal with the faced obstacles and achieve the best possible result.

We started our research by designing a questionnaire focusing on the challenges and how the teams and individuals deal with them. Survey was designed on Google for IT professionals and employees who are working for various organizations in Pakistan to be conducted online. Survey included various questions on how requirements are gathered in their firm, what are the standards used for analysis and major problems faced by them while working in globally distributed teams. We compiled all the data and analyzed it via Google forms analytics. Aim of conducting this survey via an online questionnaire was to assemble facts and figures about the root cause of the major challenges which are faced in this process of requirement gathering. We came up with the best ways to tackle these challenges and how they can be avoided.

Our target audience varied from business analysts, project managers, team leads, developers and SQA 
professionals. All the participants are currently working for Pakistan's most renowned software houses and have had ample amount of experience working in GSD environment.

Because of the varied and selected target audience, our aim was to collect at least 50 responses which is a sufficient data to analyze and conclude our research.

\section{RESUlTS OF THE QUESTIONNAIRE}

According to the online survey conducted for this research paper: as shown in Fig.1approximately $43.1 \%$ of the respondents were developers, $29.4 \%$ were SQA analyst and rest of the respondent included team leads, project managers etc.

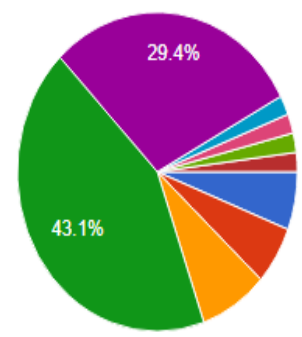

Business Analyst / Requirement
Engineer
Program Manager
Team Lead
Developer
SQA Analyst
Asst Marketing IT Advisor
Student
Freelance
Student

Fig.1. What is your role in your organization?

As shown in Fig. 2 Most of the respondents have the work experience of around 2 to 3 years such that majority was of fresh people working in the field.

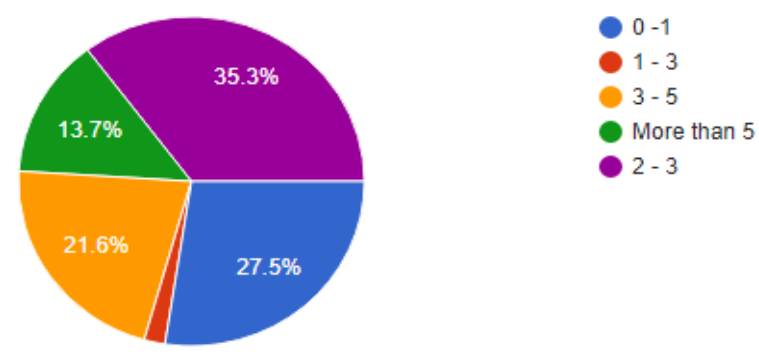

Fig.2.Year(s) of experience in related field

As shown in Fig.3 most of the respondents have worked in the globally distributed environment which helped us in analyzing our result as they would have personal experience while filling in this survey form
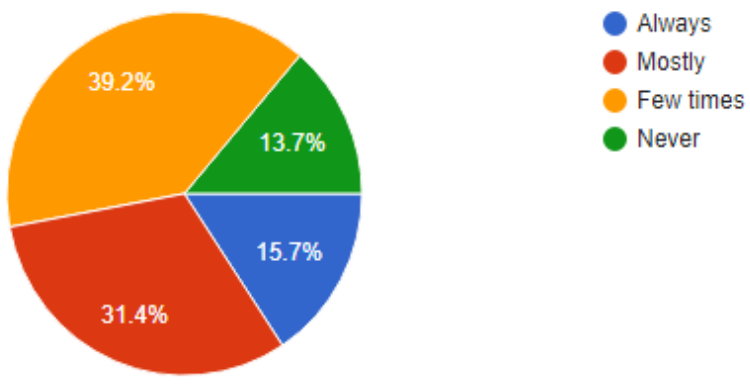

Fig.3.Have you ever worked with globally distributed team?
As shown in Fig.4 personal software process tends to have more popularity in organizations. After that approx $23.5 \%$ of the respondents said that CMMI is implemented in their organization for ensuring quality.

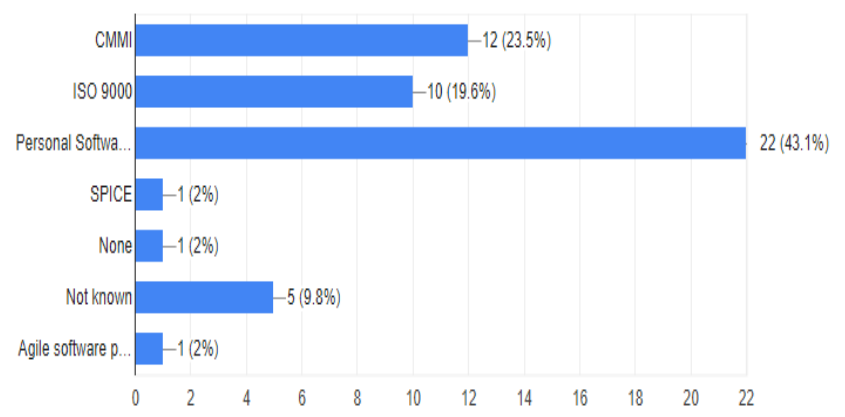

Fig.4.Which of the following technique is used in your company for ensuring quality?

As per Fig.5 however, from the results of the questionnaire it was observed that in most organizations say $37 \%$ simple work break down structure is used for the purpose of planning their project.

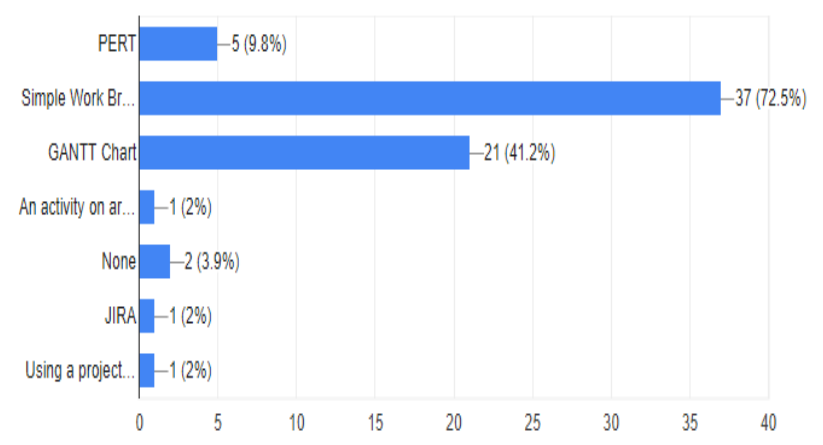

Fig.5.Your organization uses which od the following techniques(s) for project planning

As shown in Fig.6,with the boom of SCRUM methodology in recent years most of the organizations are seen to be implementing scrum. Benefits of this methodology have proven to be of real help in many cases. Scrum also facilitates with the benefit of how to handle changing requirements which is the major problem of GSD projects
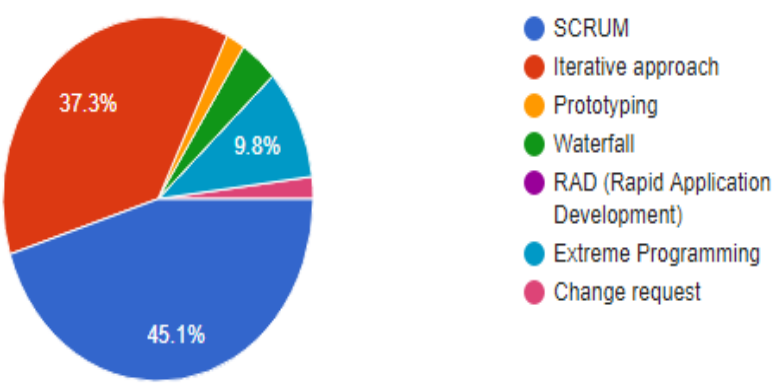

Fig.6.Which development methodology is implemented in your organization? 
As shown in Fig.7 approximately $70.6 \%$ of the respondents said that a standard process is used in their organization for gathering requirements, $20 \%$ had no idea which process is being used.
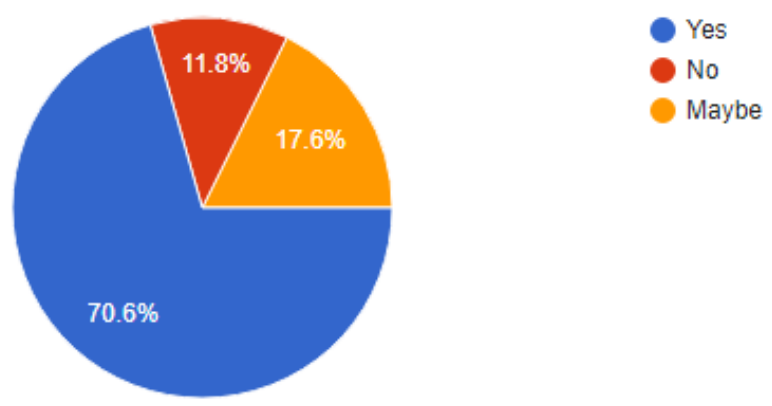

Fig.7.Does a standard process for requirement gathering is used in your organization?

Communication tool plays a very important role when teams are globally distributed. As shown in Fig.8 most of the respondents said that they use Emails for communicating with their offshore teams and clients. After that Skype is seen as the most popular mean of communication in GSD environment.

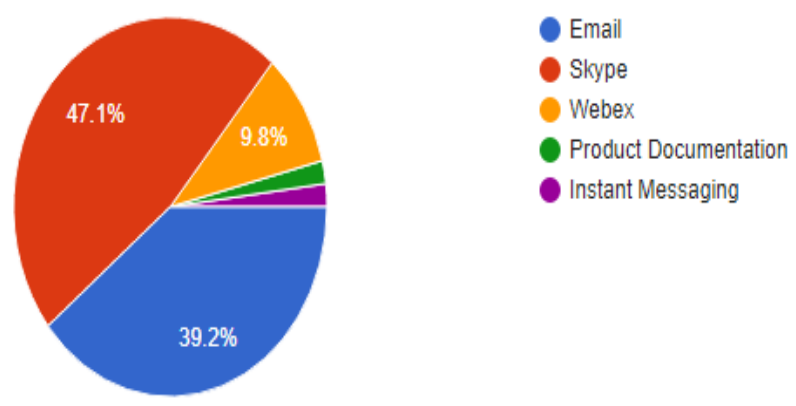

Fig.8.Which mean of communication is preferred in your organization to communicate with offshore teams?

Frequency of communication within team should be appropriate such that no communication gap occurs. Results in Fig.9 shows that approx. $47.1 \%$ of the GSD team communicate on weekly basis to discuss the status requirements during the life cycle.

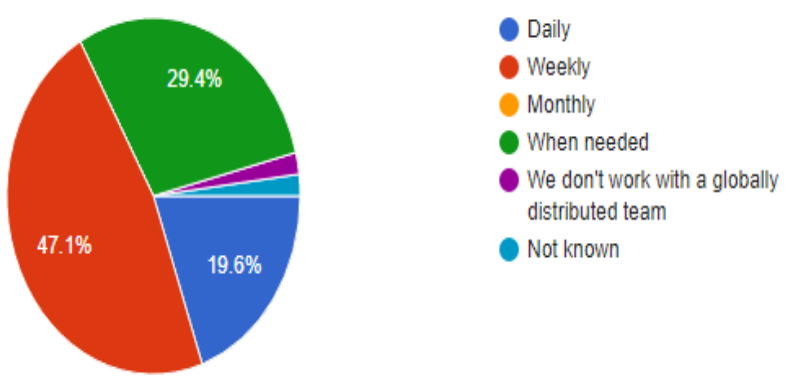

Fig.9. How frequently your offshore teams communicate with the other globally distributed members of the team?
Change in requirement is a common practice which happens during the project due to various reasons. None of the respondents claimed that requirements do not change. Nearly each one of them has faced changes $n$ requirements. This is the reason why agile is the most preferred methodology by many organizations.

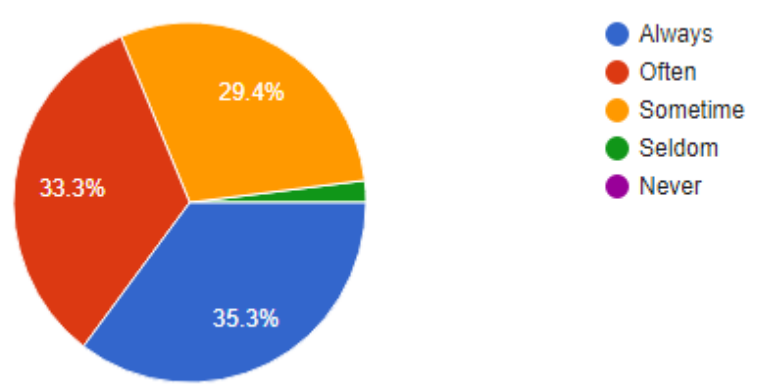

Fig.10. On average, how frequently change in gathered requirements occurs in project life cycle?

As shown in Fig.11 most of the respondents said that these changes in requirements occur because of the change in client's requirement. After that $19.6 \%$ of the respondents marked different business experience means different assumptions as being the key reason for changing requirements.

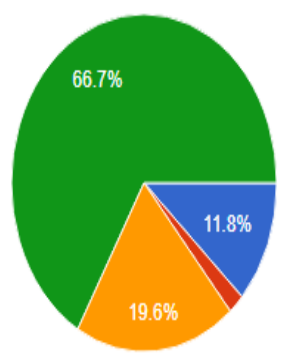

Lack of communication with client Cultural communication cifferences Different life/business experiences means different assumptions

Change in client's need

Fig.11.What're the major causes of changes in requirement?

Next we asked questions regarding how frequently respondents has faced these major challenges while working in GSD (Global Software Development) environment

As shown in Fig.12 almost all respondents had a consensus that inappropriate use of communication tool is a challenging problem in the process of gathering requirements. 


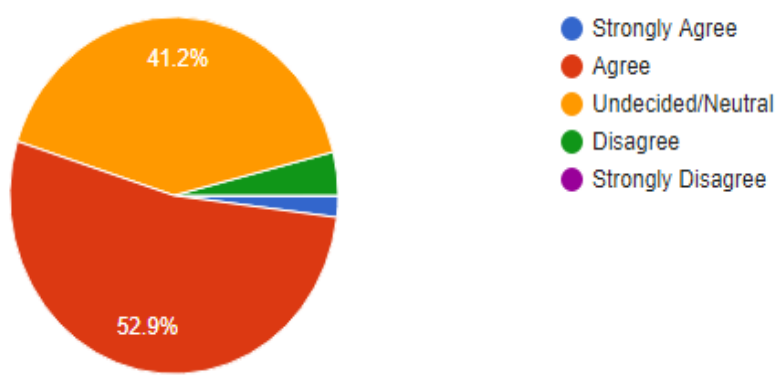

Fig.12.Inappropriate use of synchronous and asynchronous communication tools

Results in Fig. 13 shows that $45.1 \%$ of the respondents said that difficulties in learning and adopting tools which exist for GSD is a major problem faced by them. As using new tools abruptly during the project is not easy. Usually such tools have low learnability and are not much user-friendly or have attractive GUI which may increase its usability.

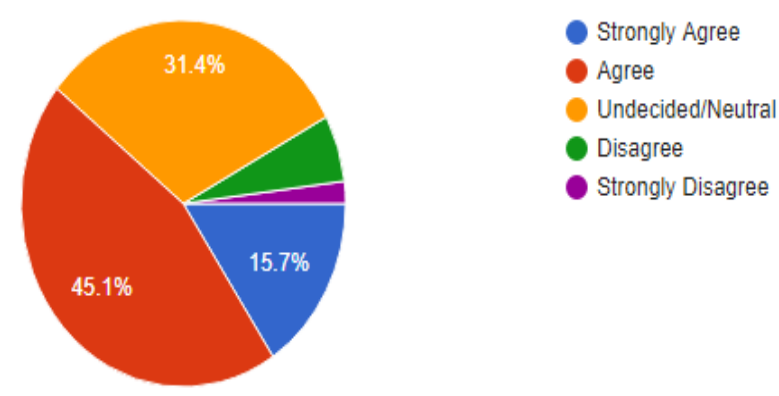

Fig.13.Difficulties in adopting and learning existing tools for GSD projects

When teams are globally distributed integration of data is to be done from time to time so that whole team can be brought on the same page. As shown in Fig.14, 35.3\% of the respondents said that they agree that lack of knowledge regarding data integration tool is a problem faced commonly.
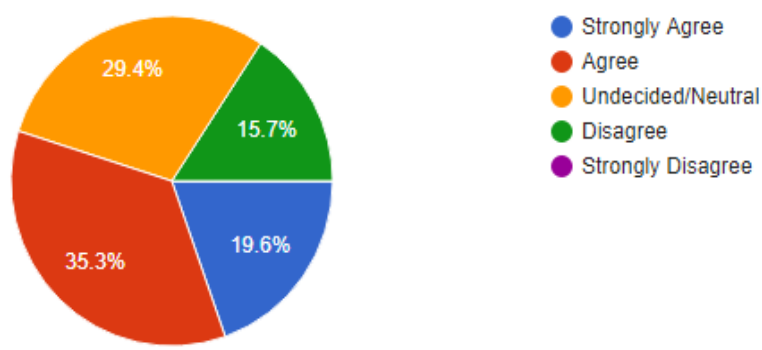

Fig.14.Lack of data integration due to different collaboration tools used in GSD projects

As shown in Fig.15 majority of the audience felt that problem with RE in GSD environment is lack of support for collaboration and in group decision making. This is really important for RE process and could be reason why people are reluctant to shift RE process to GSD environment.

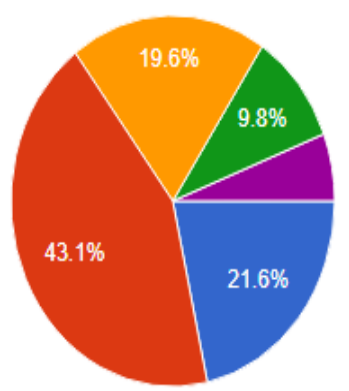

Strongly disagree

Agree

Undecided/Neutral

Disagree

Strongly agree

Fig.15.Lack of support for collaboration and group decision making

As shown in Fig.16 majority of team disagreed or had no opinion on lack of privacy and security of the GSD tools, which reflects that for teams this might not be a big problem in the shift.
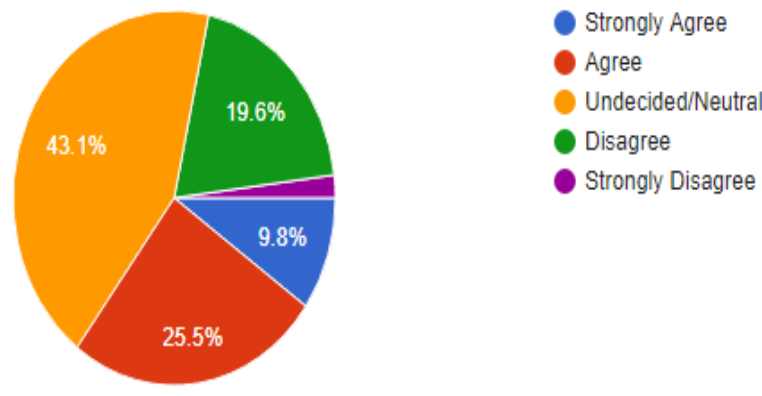

Fig.16.Lack of security and privacy in communication and collaboration tools

As shown in Fig.17 most of the respondent agreed that there are many tools available in market but they are reluctant to adopt because of lack of awareness or experience with new emerging tools.

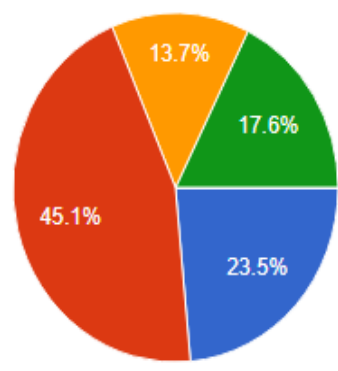

$$
\begin{aligned}
& \text { Strongly Agree } \\
& \text { Agree } \\
& \text { Undecided/Neutral } \\
& \text { Disagree } \\
& \text { Strongly Disagree }
\end{aligned}
$$

Fig.17.Lack of awareness of existing tools used in GSD projects

Fig.18 shows that most of the respondent agreed that keeping track of progress in GSD environment is an issue faced by members when working in distributed team. It's difficult for managers and team leaders to monitor and control project's progress during project life cycle. 


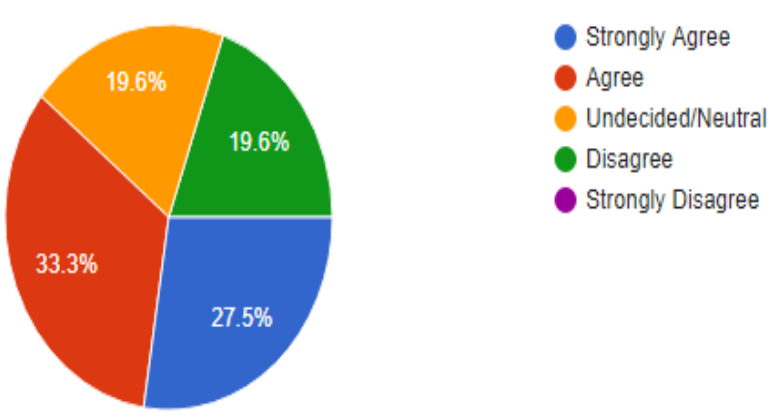

Fig.18.Lack of ability to track the progress of tasks assigned to team members GSD projects

\section{ANALYSIS AND DISCUSSION}

In this section, we have discussed the most important lessons learnt on the basis of the survey conducted and literature studied which was previously done on the discussing the challenges faced by people in requirement gathering when working in globally distributed teams.

\section{A. All Mode Of Communication Needs To Be Documented}

A good communication is needed among team members to complete a project successfully. In GSD environment teams have to primarily rely on electronic communication. To avoid any miscommunication that can harm the team trust and project outcome, the project team needs to plan out the communication in reliable and timely manner. This is especially important when team is distributed.

All communication especially synchronous i.e. communication in Live Meeting, Conference call, Audio/Video conference, Instant Messaging or texting should be recorded in some documented form for future references. Project teams usually religiously record all their communication with client but may neglect documentation for internal communications. Documentation for internal communication is really important when team is distributed in global environment. It helps all team to be on same page regarding project deliverables, scope and any changes in requirements.

\section{B. A Careful Consideration Is Needed While Selecting Tool And Techniques For Team's Communication And Collaboration}

With the increasing pool of software tools in disposal for teams, it is a challenging task to select and adopt an appropriate tool according to team setup and project needs. In most cases there is no proper procedure to select the best tool for the project. Additionally, teams are resistant to change and therefore are resistant to change the current tool with new suitable tool.

Also, due to time differences and other cultural issues, team highly relies on asynchronous tools such as Email rather than synchronous tools such Skype. Researches show that it's crucial to use rich synchronous tool to support iterative understanding in requirement engineering process.

\section{Agile Process Of Software Development Provides The Needed Flexibility In Requirement Engineering}

Because of short project lifecycle and rapid technology advancement, more software development companies have begun to elaborate requirements progressively. Agile methods both in software engineering and project management are really helpful in catering to these changes in requirements. Scrum methodology is recommended software companies who want to develop and deliver a quality software in short time. (Abeer M. AlMutairi, 2015)

Even though many distributed teams are bit reluctant to adopt agile practices across the project, even those projects who claim to adopt agile practices are not fully agile in nature but rather hybrid. But with current technologies and processes, teams can efficiently adopt agile practices to manage rapid and frequent changes. Agile processes meet both the task and relational needs for requirement engineering success.

\section{A Centralized Project Planning Techniques Needs To Be Used To Ensure Whole Team Is On Same Page}

One of the major concerns of working in GSD is to know overall project status at any phase of the project. Individual teams can provide their progress but how to evaluate project's overall status becomes issue for the managers.

A centralized project planning techniques is needed when you work in GSD environment, so that whole team is on one page. Overall project planning should be consistent across all the teams involved in the project. The individual teams can have their own individual planning techniques and tools but they need to have a process so that their planning techniques can be integrated with each other for overall project planning and monitoring.

\section{E. Tools And Techniques To Track The Progress Of Tasks Assigned To Team Members In GSD Projects Are Important.}

A project suitable tools and techniques should be adopted to keep track of progress of task assigned to the team member as well as to monitor the project overall status. Techniques such as Kanban System should be adopted where managers and leaders can track each member assigned tasks status. There are many tools in market which can cater to teams working in GSD environment. These tools should be able to give teams a way to track their work, so that everyone in the teams knows who's doing what, by when. These interfaces should be easy and simple to use to collaborate with teams and client.

However, the communication between GSD teams can be improved by developing a web application (Distributed Scrum Web Application) which may help teams to smooth the progress of their work. (M. Rizwan Jameel Qureshi, 2014) 


\section{F. Run The Weekly Team Meeting (Every Week).}

Weekly meetings are the driving force in building your project and developing better and newer ways to complete the project to success. This also helps to keep the project going smoothly and heightens the productivity, by setting goals together and striving to hit these goals by next meeting.

This is especially important in large and distributed teams, where these meetings not only help them by providing one platform to collaborate together but also helps in team building. This also gives manager another way to get to know the individual progress of the team members.

G. Client Representative That Facilitate Requirement Engineering Processes Have More Impact On The Project Outcome As Compared To The Project Team Representatives Involved.

The presence and active client representative has a direct positive impact on the requirement engineering process. Their presence and timely involvement in requirement process can help to enhance project quality. They can clarify and streamline requirement discrepancies. They can also help with apportion work to developers and, to reduce any confusion and misunderstandings. This also saves teams time in decision making on spot and also reduces the chances wrong assumptions by the team.

H. Presence Of Experienced Client Representative During The Requirements Phase Leads To A More Successful Project.

Client representative are only beneficial for requirement phase when they are experienced in the specific domain and have enough knowledge to explain project needs to team. They need to ensure that team's efforts are aligned with their needs. They should have some authority to make at least minor decisions as a client decisions.

\section{There Need To Be A Shared Understanding Between Teams About How Requirements Changes Would Be Dealt With.}

Requirements constantly evolve either due to market trends or sometime needs of client. Awareness of change process implemented will affect in converting vague requirements into more clear and understandable (Munawar Hayat, 2015). Like all other process, all teams should be on the same page on how the requirement changes will be dealt with. This should be clear from the beginning of the project. It's helpful to keep project tasks separate from feature requests. There should be a proper change approval process and a log should be maintained to document every change. Feature creeps are mostly due to lack of or unclear project planning and lack of access to documentation. By using online collaboration tools team ensure the all project team members and stakeholder can access up to date project information, allowing them to make decisions accordingly.

\section{CONCLUSION}

Global Software development is widely adapted by organization around the world, but we see that most of organization has only adopted this for development and testing phases. Organizations are reluctant to bring RE process in GSD environment because as ineffective requirement engineering process may sabotage whole project. Also, organizations have realized that RE process is phase of communication and wants to make it as smooth as possible. Even though RE in GSD environment can be complex but with technologies and researches on our disposal, we can now try to shift this process in GSD environment too. Teams need to spend some significant efforts in early stages to decide on tools, controlling and monitoring process to fully adopt GSD all over project phase including RE.

The proposed solution in the paper, are few of the best practices that teams can adopt and alter according to their project needs to work best in GSD environment.

\section{REFRENCES}

[1] Ali, N. (2015). A Method of requirements change management for global software development. Information and Software Technology 70 .

[2] Ali, N. (2015). Managing requirements change in Global Software Development.

[3] Ali, S. (2016). An Improved Framework for Requirement Implementation in the context of Global Software Development: A Systematic Literature Review Protocol. International Journal of Modern Nonlinear Theory and Application, 161-170.

[4] de Gea, C. J. (2016). Co-located and distributed natural-language requirements specification: traditional versus reuse-based techniques. Co-located and distributed natural-language requirements specification: traditional versus reuse-based techniques.

[5] Fernández, D. M. (2013). Naming the Pain in Requirements Engineering. Germany: ISERN 2012.

[6] Khan, H. H. (2014). Factors generating risks during requirement engineering process in global software development environment. International Journal of Digital Information and Wireless Communications (IJDIWC), (pp. 63-78).

[7] Khan, H. H. (2013). Risk Generating Situations of Requirement Engineering in Global Software Development. Second International Conference on Informatics Engineering \& Information Science (ICIEIS2013), (pp. 221-233).

[8] Kumari, S. N. (May 2013). A survey on global requirements elicitation issues and proposed research framework. . In Software Engineering and Service Science (ICSESS), 2013 4th IEEE International Conference, (pp. 554-557).

[9] Minhas, N. M. (2014). An Improved Framework for Requirement Change Management in Global Software Development. Journal of Software Engineering and Applications, 779-790.

[10] Minhas, N. M. (2014). An improved framework for requirement change management in global software development. Journal of Software Engineering and Applications, 7(9), 779, (pp. 7(9), 779).

[11] Niazi, M. M. Challenges of the Existing Tools Used in Global Software Development Projects. Department of 
Information and Computer Science, King Fahd University of Petroleum and Minerals, Saudi Arabia.

[12] Rahman, M. \&. (2015). MITIGATION APPROACHES FOR COMMON ISSUES AND CHALLENGES WHEN USING SCRUM IN GLOBAL SOFTWARE DEVELOPMENT

[13] Yadav, V. A. (2016). Considerations for Effective Requirements Analysis in Offshore Software Development Projects: Lessons from Multi-method Research. Communications of the Association for Information Systems., (pp. 39(1), 11.).

[14] Yadav, V. (2016). Considerations for Effective Requirements Analysis in offshore software development projects: Lessons from multi-method research. Communications of the Association for Information Systems , 188-213.

[15] Yaseen, M. A. (2016). An Improved Framework for Requirement Implementation in the context of Global Software Development: A Systematic Literature Review Protocol. International Journal of Database Theory and Application, (pp. 9(6), 161-170.).

[16] Abeer M. AlMutairi, M. R. (2015). The Proposal of Scaling the Roles in Scrum of. MECS (p. 7). Jeddah, Saudi Arabia: I.J. Information Technology and Computer Science.

[17] M. Rizwan Jameel Qureshi, N. A. (2014). Mitigating Coordination Costs in Global. I.J. Information Engineering and Electronic Business (pp. 16-21). Jeddah, Saudi Arabia: MECS.

[18] Munawar Hayat, M. R. (2015). Measuring the Effect of CMMI Quality Standard. I.J. Information Engineering and Electronic Business (pp. 46-52). Lahore, Pakistan: MECS.

\section{Authors' Profiles}

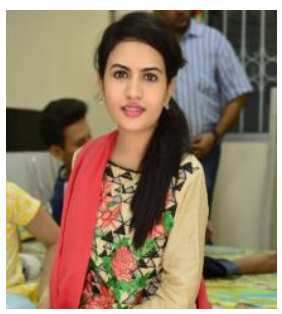

Nabiha Usmani: Born and raised in Karachi, Pakistan. Nabiha has done her Bachelor's in Computer Science from University of Karachi and is currently enrolled in a Masters program in Institute Of Business Administration.

After graduating from KU in 2014 she started working as Software quality Assurance Engineer in Matrix systems her job description also includes the responsibilities of a
Business Analyst. She holds an experience of around 2 years in the related field. Recently her paper got published in IBIMA $28^{\text {th }}$ conference named "Benefits to Organization after migrating to Scrum".

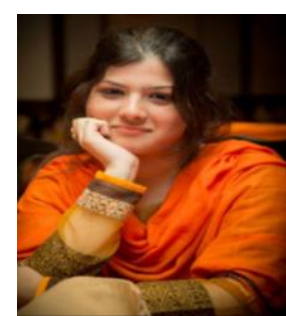

Rabbia Hassan: Rabbia holds a Bachelor's degree in Computer Science from NUCES (FAST) University, Karachi, and currently perusing her MS degree from IBA, Karachi. She had always been fascinated with computer programming and these educational institutes have helped her shone her skills where she discovered her interest in Software Engineering, distributed and concurrent systems and mobile development.

After graduating from FAST in June 2013, she joined IHS in September 2013 as a Software Engineer. At IHS, she currently works on Java based mobile and web projects. She aspires to learn and work on new and emerging technologies in future.

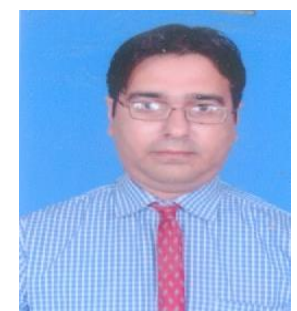

Waqas Mahmood: Waqas has done MS in Economics and Finance from IoBM (CBM), Karachi in 2012. Prior to that Waqas did MS in Software Project Management from NUCES (FAST) in 2010. Moreover he holds Masters of Engineering (M.Engg) degree from Hamdard University and M.E degree from NED. He completed his BS (Engg) from Sir Syed University of Engineering \& Technology in 1998.

From January 2008 till present he has been working as a Joint Director in State Bank of Pakistan. He has been a part of IBA's visiting faculty from past 10 years.

How to cite this paper: Nabiha Usmani, Rabbia Hassan, Waqas Mahmood," Impediments to Requirement Engineering in Distributed Team", International Journal of Information Engineering and Electronic Business(IJIEEB), Vol.9, No.6, pp. 10-18, 2017. DOI: 10.5815/ijieeb.2017.06.02 